\title{
Precision measurements of Standard Model parameters and Re- view of Drell-Yan and vector boson plus jets measurements with the ATLAS detector
}

\author{
Noemi Calace ${ }^{1, a}$, on behalf of the ATLAS Collaboration \\ ${ }^{1}$ Département de physique nucléaire et corpusculaire, Section de Physique, Université de Genève, 24 Quai \\ Ernest-Ansermet, Genève, Switzerland
}

\begin{abstract}
The inclusive productions of the $W$ and the on- or off-shell $Z / \gamma^{*}$ bosons are standard candles at hadron colliders, while the productions of light and heavy-flavour jets in association with a $W$ or a $Z$ boson are important processes to study quantum-chromodynamics (QCD) in multi-scale environments. The measurements of their production cross-sections integrated and differential in several variables have been measured at 7 and $8 \mathrm{TeV}$ centre-of-mass energies and are compared to high-order QCD calculations and Monte Carlo simulations. These measurements have an impact on our knowledge of the parton densities of the proton, and test soft resummation effects and hard emissions for small and large momentum transfers and in multi-scale processes. Precision measurements of fundamental Standard Model parameters in Drell-Yan final states are also performed to describe the angular distributions of the decay lepton.

Run-1 studies carried out by the ATLAS Collaboration are re-viewed and first LHC Run-2 results are included.
\end{abstract}

\section{Introduction}

The ATLAS Collaboration [1] has carried out multiple measurements of vector boson production both inclusively and with associated light or heavy flavour jets at the Large Hadron Collider (LHC). Thanks to their large production cross section, events containing a $W$ or a $Z$ boson are produced abundantly at the LHC. These events provide the ideal test to probe high-order quantum-chromo-dynamics (QCD), measure electro-weak parameters and constrain parton distribution functions (PDFs). In the last years, these measurements have reached high experimental precision allowing detailed comparisons with theoretical predictions at next-to-next-to-leading-order (NNLO) QCD. Moreover, the dynamical effects of the strong interaction can be probed by measuring QCD radiation in the different kinematic ranges: the high and low vector boson $p_{\mathrm{T}}$ regimes can probe high-order QCD corrections and resummation techniques, respectively.

The studies of $W$ or $Z$ boson events where accompanying jets are explicitly reconstructed, either inclusively in jet flavour or exclusively as heavy-flavour jets, provide precision tests of high-order QCD calculations and can also be used to constrain PDFs. Furthermore, such processes constitute an important background to Standard Model (SM) as well as new physics searches.

a e-mail: noemi.calace@ cern.ch 
The measurements presented here are based on the data collected by the ATLAS detector during the LHC Run-1 proton-proton collision running at $7 \mathrm{TeV}$ and $8 \mathrm{TeV}$ centre-of-mass energy, including also the first LHC Run-2 results at $13 \mathrm{TeV}$ centre-of-mass energy.

\section{Forward-backward asymmetry of electron and muon pair-production}

The vector and axial-vector couplings in the neutral current annihilation process $q \bar{q} \rightarrow Z / \gamma^{*} \rightarrow l^{+} l^{-}$ lead to a forward-backward asymmetry $A_{\mathrm{FB}}$ in the polar angle distribution of the final state lepton $l^{-}$ with respect to the quark direction in the rest frame of the dilepton system. The ATLAS experiment has measured the forward-backward asymmetry in electron and muon pairs from $Z / \gamma^{*}$ boson decays and extracted the weak mixing angle [2] based on the full set of proton-proton $(p p)$ collision data collected in 2011 at the LHC at a centre-of-mass energy of $\sqrt{s}=7 \mathrm{TeV}$, corresponding to an integrated luminosity of $4.8 \mathrm{fb}^{-1}$.

The data are analysed over a range of dilepton invariant masses from $66 \mathrm{GeV}$ to $1000 \mathrm{GeV}$ in the central-central electron (where both electrons are reconstructed in the central region of the detector $|\eta|<2.47$ ) and muon channels, and up to $250 \mathrm{GeV}$ in the central-forward electron channel. The latter includes events where one electron is reconstructed in the forward pseudorapidity range $(2.5<|\eta|<$ 4.9). The forward-backward asymmetry is measured separately for the three channels as a function of the dilepton invariant mass and unfolded for detector effects and final-state radiation. An additional unfolding procedure is used to further correct the observed values of $A_{\mathrm{FB}}$ to remove dilution effects, which occur when the wrong choice is made for the direction of the quark. The resulting $A_{\mathrm{FB}}^{\text {cor }}$ values are found to be in agreement with the corresponding Standard Model predictions. The final $A_{\mathrm{FB}}^{\text {cor }}$ values as a function of the dilepton invariant mass for the central-central electron, central-forward electron and muon channels are shown in Figure 1.

Several SM parameters can be extracted from the dependence of the $A_{\mathrm{FB}}$ values on the invariant dilepton mass. One of these is the electroweak mixing angle $\sin ^{2} \theta_{\text {eff }}^{\text {lept }}$, also related to the effective leptonic weak mixing angle $\sin ^{2} \theta_{\mathrm{eff}}^{\text {lept }}$. The value of the $A_{\mathrm{FB}}$ at the peak of the $Z / \gamma^{*}$ resonance, $A_{\mathrm{FB}}^{0, l}$, can be also written as a function of the asymmetry parameters $A_{l}$ and $A_{q}$,

$$
A_{\mathrm{FB}}^{0, l}=\frac{3}{4} A_{l} A_{q}
$$

with $l(q)$ denoting the leptons (quarks) in the final (initial) state and with the parameters $A_{l}$ and $A_{q}$ directly related to the electroweak vector and axial-vector couplings.

In the analysis the effective weak mixing angle $\left(\sin ^{2} \theta_{\text {eff }}^{\text {lept }}\right)$ and the muon asymmetry parameter $A_{\mu}$ are extracted from the forward-backward asymmetry measurements $A_{\mathrm{FB}}$.

For the effective weak mixing angle, the results from the electron and muon final states are combined:

$$
\sin ^{2} \theta_{\mathrm{eff}}^{\text {lept }}=0.2308 \pm 0.0005 \text { (stat.) } \pm 0.0006 \text { (syst.) } \pm 0.0009(\mathrm{PDF})=0.2308 \pm 0.0012 \text { (tot.) }
$$

The dominant uncertainty comes from knowledge of the PDFs.

The result from the muon channel, when converted to the asymmetry parameter $A_{\mu}$, yields

$$
A_{\mu}=0.153 \pm 0.007 \text { (stat.) } \pm 0.006 \text { (syst.) } \pm 0.007 \text { (PDF) }=0.153 \pm 0.012 \text { (tot.), }
$$

where the uncertainties are propagated from the uncertainties on $\sin ^{2} \theta_{\text {eff }}^{\text {lept }}$. The measurement is in good agreement with the best previous ones. 


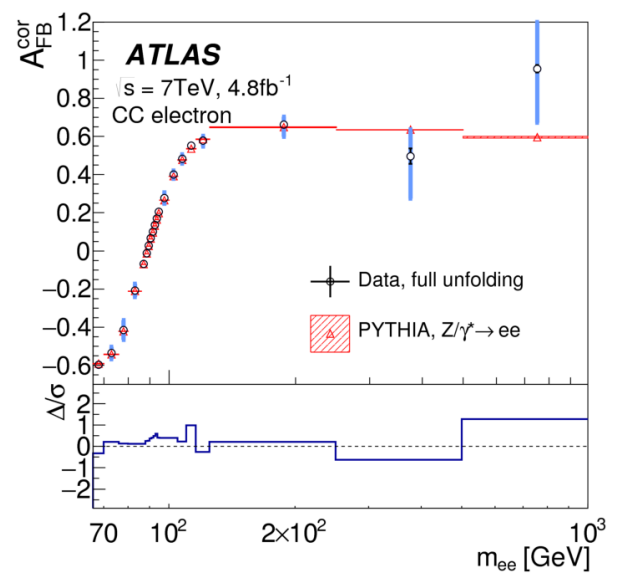

(a)

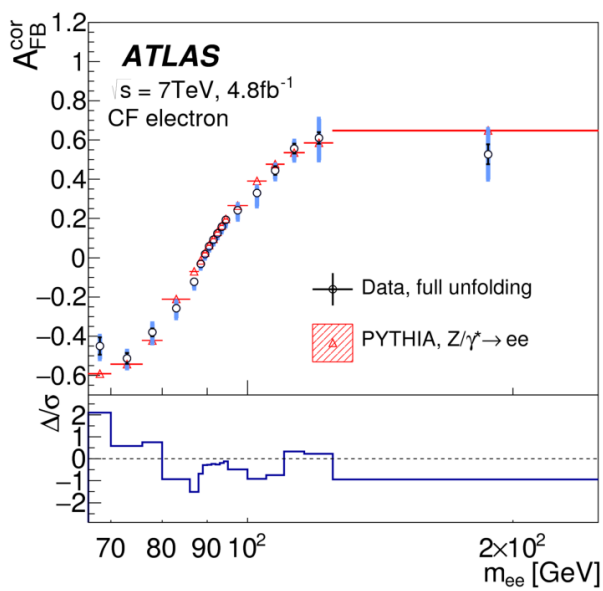

(b)

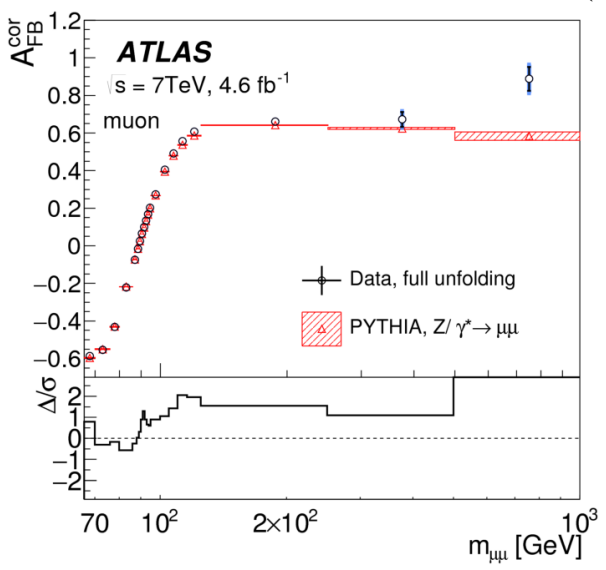

(c)

Figure 1: Forward-backward asymmetry $\left(A_{\mathrm{FB}}^{\text {cor }}\right)$ values as a function of the dilepton invariant mass for the (a) central-central, electron, (b) central-forward electron and (c) muon channels. Leptons are unfolded to Born level to account for mass bin migration and dilution effects are corrected. The measurement is extrapolated to the full phase space, and the results are compared to truth-level MC information. For the data, the black inner error bars represent the statistical component and the lighter outer error bars the total error (statistical and systematic added in quadrature). The boxed shaded regions for the $\mathrm{MC}$ expectations represent only the statistical uncertainty; theoretical uncertainties are included in the data systematic uncertainties. The lower panel of each plot shows the pull value, $\Delta / \sigma$, for each mass bin, where $\Delta$ is the difference between data and simulation and $\sigma$ is the sum in quadrature of the data and simulation uncertainties. Taken from Ref. [2].

\section{$3 Z / \gamma^{*}$ transverse momentum and $\phi_{\eta}^{*}$}

The measurements of boosted $Z$-boson production, i.e. with non-zero $p_{\mathrm{T}}$, can be used to constrain theoretical models of perturbative QCD (pQCD). The low $Z-p_{\mathrm{T}}$ region can probe resummation tech- 
niques whereas the high $Z$ - $p_{\mathrm{T}}$ regime probes higher-order QCD corrections. The measurement is an important input to constrain the parton shower parameters of MC generators and provide a better understanding of the Higgs boson production. A correct modelling of the vector boson $p_{\mathrm{T}}$ spectrum is crucial for many physics analyses, e.g. the $W$ mass measurement. Using $p p$ collision data at a centreof-mass energy of $\sqrt{s}=7 \mathrm{TeV}$ at the LHC, the ATLAS collaboration performed the measurements of the $Z / \gamma^{*}$ boson transverse momentum spectrum, in the $Z / \gamma^{*} \rightarrow e^{+} e^{-}$and $Z / \gamma^{*} \rightarrow \mu^{+} \mu^{-}$channels. Normalised differential cross sections as a function of the $Z / \gamma^{*}$ boson transverse momentum are measured for transverse momenta up to $800 \mathrm{GeV}$. The measurement in performed inclusively up to $Z / \gamma^{*}$ rapidities of 2.4 , as well as in three rapidity bins [3]. The cross-section measurements are then compared to $\mathrm{pQCD}$ and resummed predictions.

Figure $2 \mathrm{a}$ shows the ratio of the measured cross section as a function of the boson transverse momentum with predictions from Fewz [4-6] and DynnLo [7, 8]. The large discrepancy at low $p_{\mathrm{T}}$ is due to the not inclusion of resummation effects. In Figure $2 b$, the measurement is compared to ResBos $[9,10]$ which is in agreement within the estimated uncertainties at low $p_{\mathrm{T}}$, as it includes resummation effects. However, the precision of direct measurements of the $Z / \gamma^{*}$ spectrum at low $p_{\mathrm{T}}$ at the LHC is limited by the experimental resolution and systematic uncertainties: the angular correlations of the lepton pair, $\phi_{\eta}^{*}$, can probe the low $p_{\mathrm{T}}$ domain providing a better experimental resolution and a smaller sensitivity to experimental systematic uncertainties [11]. As it is defined [12], $\phi_{\eta}^{*}$ depends exclusively on the directions of the two lepton tracks, which are better measured than their momenta.

The $Z / \gamma^{*} \rightarrow e^{+} e^{-}$and $Z / \gamma^{*} \rightarrow \mu^{+} \mu^{-}$decays produced in $p p$ collisions at a centre-of-mass energy of $\sqrt{s}=7 \mathrm{TeV}$ are used for this measurement. The data were collected with the ATLAS detector at the LHC and correspond to an integrated luminosity of $4.6 \mathrm{fb}^{-1}$. Normalised differential cross sections as a function of $\phi_{\eta}^{*}$ are measured separately for electron and muon decay channels. These channels are then combined for improved accuracy. The results are compared to QCD calculations and to predictions from different Monte Carlo event generators.

The best descriptions of the measured $\phi_{\eta}^{*}$ spectrum are provided by SHERPa and Powheg+Pythia8 MC event generators (Figure 3). For $\phi_{\eta}^{*}$ values above 0.1, predictions from SHERPA are able to reproduce the data to within $\sim 2 \%$. The low $\phi_{\eta}^{*}$ part of the spectrum is, however, described less accurately than by ResBos.

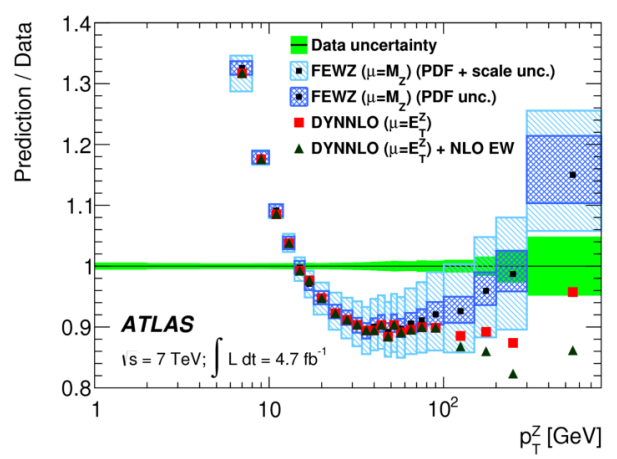

(a)

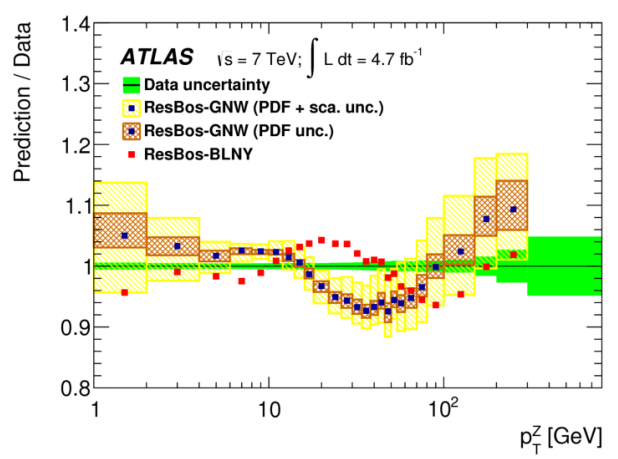

(b)

Figure 2: Comparison of the $\mathrm{Z}$ boson $p_{\mathrm{T}}$ distributions predicted by different computations: Fewz and Dynnlo (a) and ResBos (b) with the Born-level combined measurement. Taken from Ref. [3]. 


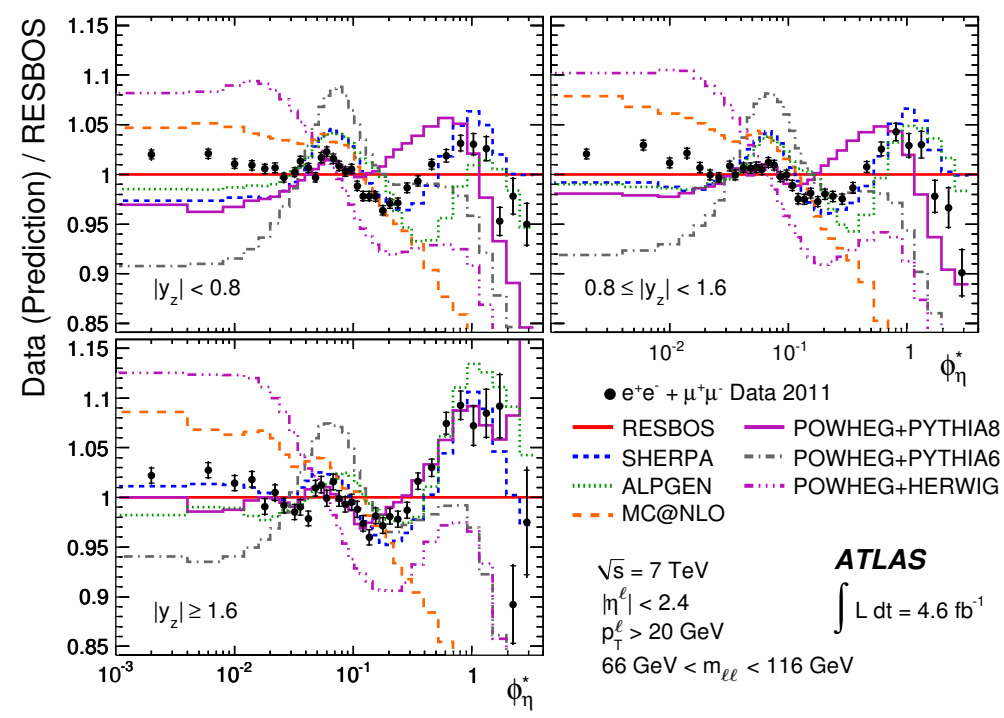

Figure 3: The ratio of the combined normalised differential cross section $1 / \sigma^{\mathrm{fid}} \cdot d \sigma^{\mathrm{fid}} / d \phi_{\eta}^{*}$ to the ResBos predictions as a function of $\phi_{\eta}^{*}$ in three ranges of $\left|y_{z}\right|$. The inner and outer error bars on the data points represent the statistical and total uncertainties, respectively. The uncertainty due to quantum-electrodynamic first-state radiation is included in the total uncertainties. The measurements are also compared to predictions from different MC event generators. Taken from Ref. [11].

\section{$4 W+$ jets measurement}

$W$ boson plus jets production has been measured in proton-proton collisions at $\sqrt{s}=7 \mathrm{TeV}$ with the ATLAS experiment [13]. Final states with up to seven jets are measured, with comparisons to next-to-leading-order (NLO) QCD predictions for up to five jets. With an integrated luminosity of 4.6 $\mathrm{fb}^{-1}$, the data set allowed an exploration of a large kinematic range, including jet production up to a transverse momentum of $1 \mathrm{TeV}$ in a large rapidity acceptance region $|\mathrm{y}|<4.4$.

At these high transverse momenta higher-order electroweak effects can become as important as those from pQCD corrections. The cross section has been measured inclusively and differentially in several observables. Figure 4 shows the cross section for the production of $W+$ jets as a function of the inclusive jet multiplicity. The measured cross section is compared to a number of theoretical predictions at both leading-order (LO) and NLO in pQCD. The leading-order predictions are using AlPGEN [14], SHERPA $[15,16]$ and HEJ $[17,18]$. The NLO predictions are obtained using MEPS@ NLO [15, 16] and BlackHat+Sherpa [19-21]. All agree within the assigned experimental uncertainties. Alpgen and SHERPa show some opposite trend towards higher jet multiplicities.

\section{$5 R_{\text {jets }}$ measurement}

The $R_{\text {jets }}$ mesurement, defined as the ratio $R_{\text {jets }}=\sigma(W+$ jets $) / \sigma(Z+$ jets $)$, directly probes the difference between the kinematic distributions of the jet system recoiling against the $W$ or $Z$ bosons. In comparison to separate $W+$ jets and $Z+$ jets cross section measurements, the $R_{\text {jets }}$ measurement is a more precise test of $\mathrm{pQCD}$, since some experimental uncertainties and effects from non-perturbative 

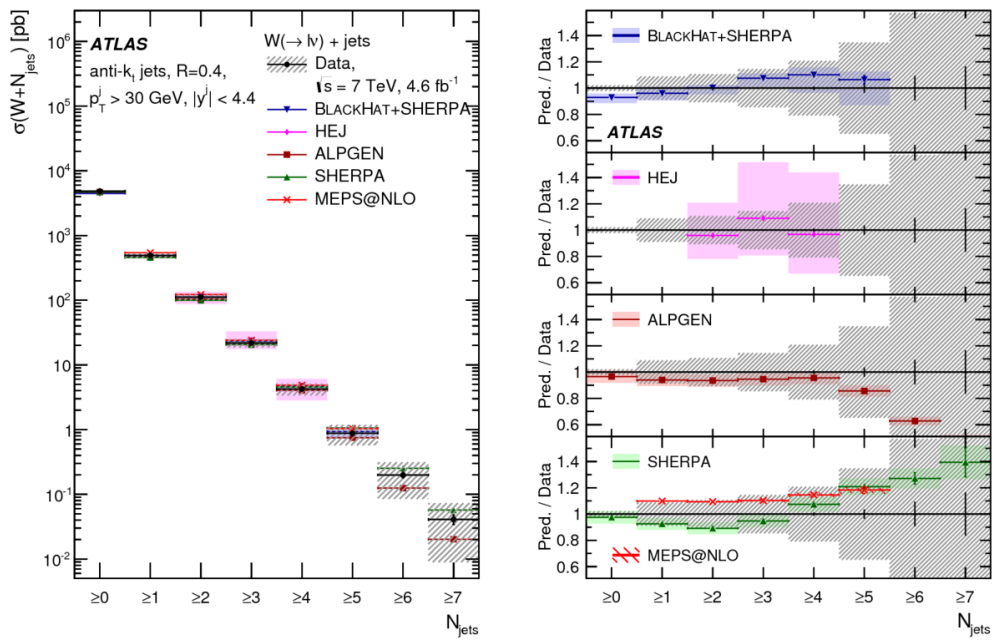

Figure 4: Cross section for the production of $W+$ jets as a function of the inclusive jet multiplicity. For the data, the statistical uncertainties are shown by the vertical bars, and the combined statistical and systematic uncertainties are shown by the black-hashed regions. The data are compared to predictions from Blackhat+Sherpa, HEJ, Alpgen, SHERPA and MEPS@NLO. The left-hand plot shows the differential cross sections and the right-hand plot shows the ratios of the predictions to the data. The theoretical uncertainties on the predictions are described in the text. Taken from Ref. [13].

processes, such as hadronization and multi-parton interactions, are greatly reduced in the ratio. This allows precise comparisons with state-of-the-art Monte Carlo simulations and NLO pQCD calculations to be made. $7 \mathrm{TeV} p p$ collisions are used to perform this measurement [22].

The $R_{\text {jets }}$ measurement is done for the electron and muon decay channels of the $W$ and $Z$ bosons for jets with transverse momentum $p_{\mathrm{T}}>30 \mathrm{GeV}$ and rapidity $|\mathrm{y}|<4.4$. The measurements of the electron and muon channels are performed in slightly different phase spaces and combined in a common phase space. An extensive set of exclusive and inclusive differential measurements have been measured and compared to a variety of theoretical predictions.

Figure 5 shows $R_{\text {jets }}$ as a function of the inclusive and exclusive jet multiplicity $\left(N_{\text {jets }}\right)$ up to four jets. The ratio is compared to a prediction calculated with BLACKHAT+SHERPA and the prediction of the Alpgen and ShERPa generators. Small deviations can be seen at larger $N_{\text {jets }}$ for the comparison with SHERPA.

\section{Run-2 results}

After a two year long shut down, ATLAS started to take data from $p p$ collision data a centre-ofmass energy of $\sqrt{s}=13 \mathrm{TeV}$. The study of leptonic $W$ and $Z$ boson decays is ideal for validating the early detector performance, such as lepton reconstruction, and the analysis software chain.

\section{1 $W$ and $Z$ boson production cross sections}

$W$ and $Z$ boson production cross sections have been measured by the ATLAS experiment with $p p$ collisions corresponding to an integrated luminosity of approximately $85 \mathrm{pb}^{-1}$, collected at $\sqrt{s}=13$ 


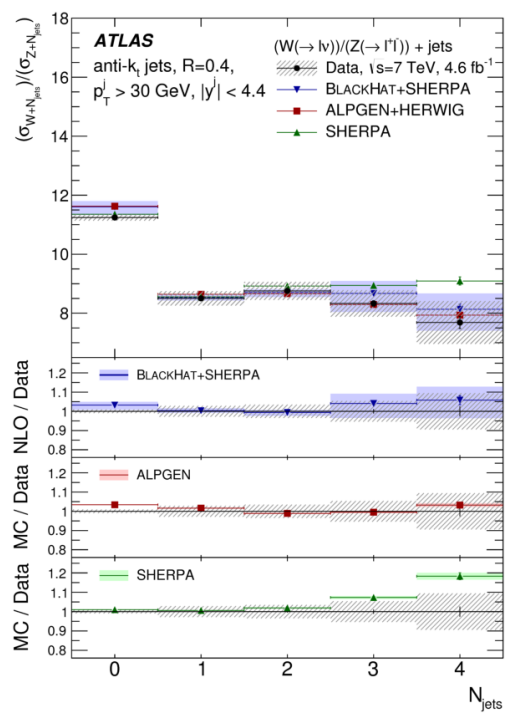

(a)

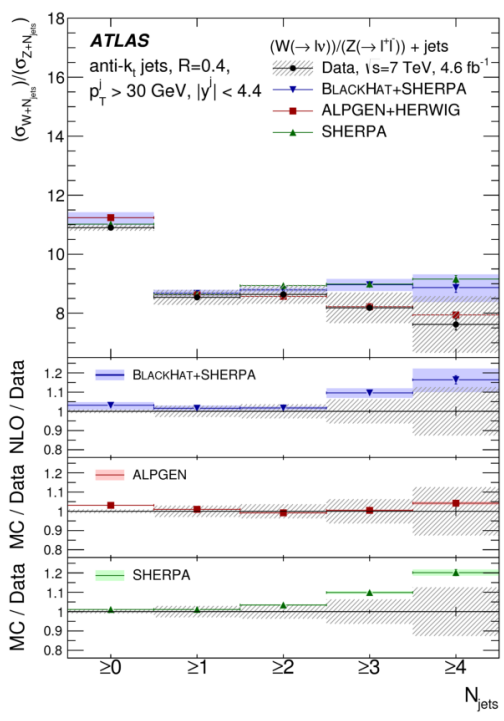

(b)

Figure 5: The ratio of $W+$ jets and $Z+$ jets production cross sections, $R_{\text {jets }}$, as a function of exclusive jet multiplicity, $N_{\text {jets }}$, (a) and inclusive jet multiplicity (b). Ratios of the BLAckHaT+SHERPA NLO calculation and the ALPGEN and Sherpa generators to the data are shown in the lower panels. Vertical error bars show the respective statistical uncertainties. The hatched error band shows statistical and systematic uncertainties added in quadrature for the data. The solid error bands show the statistical uncertainties for the AlPGEN and SHERPa predictions, and the combined statistical and theoretical uncertainties for the BцAскHAT+SHERPA prediction. Taken from Ref. [22].

$\mathrm{TeV}$ [23]. In particular, the total inclusive production cross sections for the $W \rightarrow e v, W \rightarrow \mu \nu$, $Z \rightarrow e^{+} e^{-}, Z \rightarrow \mu^{+} \mu^{-}$processes, the charge-specific cross sections for $W^{+}$and $W^{-}$boson production and the cross-section ratios of $W^{+}$to $W^{-}$production and of $W$ to $Z$ production have been measured. Figure 6a shows the dilepton mass distribution after a $Z \rightarrow e^{+} e^{-}$selection. In Figure $6 \mathrm{~b}$ the $W$ boson transverse mass for the $W \rightarrow \mu v$ channel selection is shown. The data for both selections are in good agreement with the expectation. The systematic uncertainties shown are obtained using very early studies as well as extrapolation from the Run-1 uncertainties.

The cross section results in the electron and muon channel are then combined taking into account correlated systematic uncertainties. The combined fiducial cross sections are compared in Figure 7 to the predictions which are calculated using different PDF sets. The measurements agree well with the predictions. The measurement experimental precision is already comparable to PDF uncertainties; however, the experimental precision is diluted by the uncertainty of the preliminary luminosity calibration.

The ratios of the combined fiducial cross sections for $W^{+}$to $W^{-}$boson production and for $W$ to $Z$ boson production are compared to theory predictions in Figures $8 \mathrm{a}$ and $8 \mathrm{~b}$. For the ratio $R_{W^{+} / W^{-}}=\sigma_{W^{+}}^{f i d} / \sigma_{W^{-}}^{\text {fid }}$, there is a significant scatter for different PDF predictions and the accuracy of the experimental result is comparable to the spread among them. For the ratio $R_{W / Z}=\sigma_{W}^{\mathrm{fid}} / \sigma_{Z}^{\mathrm{fid}}$, the predictions agree within quoted uncertainties and the measurement is consistent with all of them. 


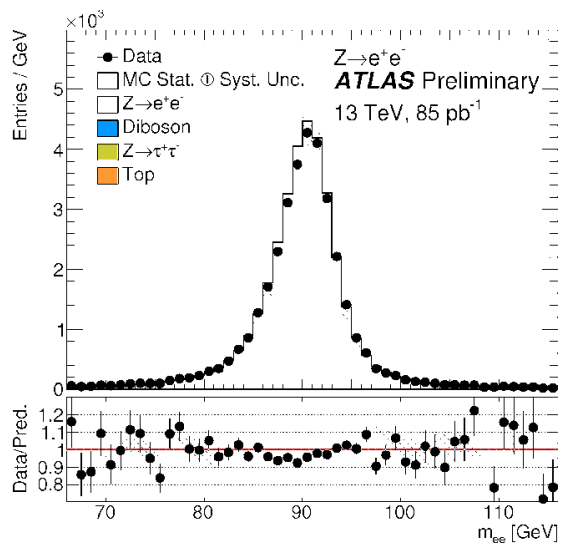

(a)

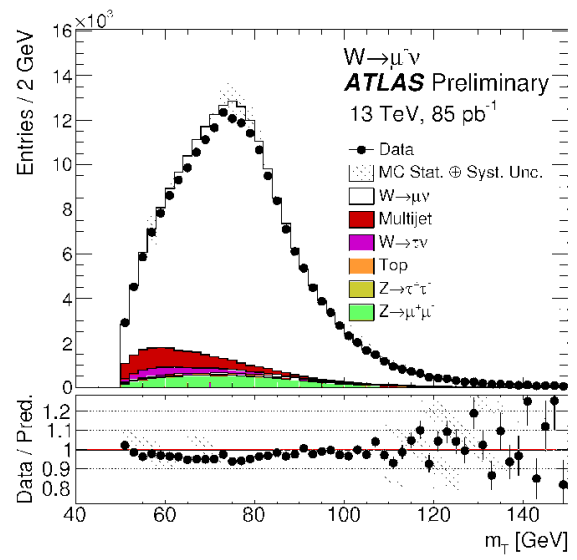

(b)

Figure 6: Dilepton mass distribution after the $Z \rightarrow e^{+} e^{-}$selection (a) and transverse mass distribution, calculated from the lepton and the missing transverse energy $E_{\mathrm{T}}^{\text {miss }}$ from $W \rightarrow \mu v$ selection (b). The expected contributions from all backgrounds are estimated with Monte Carlo simulations, except for the multi-jet background in the $W$ boson selection which is estimated with a data-driven method. Systematic uncertainties for the signal and background distributions are combined in the shaded band, the statistical uncertainty is shown on the data points. The luminosity uncertainty is not included. Taken from Ref. [23].

\section{$6.2 Z+$ jets measurement}

Using data corresponding to an integrated luminosity of $85 \mathrm{pb}^{-1}$ at $13 \mathrm{TeV}$, preliminary measurements of the cross section for the production of a $Z$ boson in association with jets have been performed by the ATLAS collaboration [24].

The $Z$ boson is identified using its decays to electron and muon pairs $\left(Z \rightarrow e^{+} e^{-}, Z \rightarrow \mu^{+} \mu^{-}\right)$ produced in association with one or more jets in the kinematic range of $p_{\mathrm{T}}^{\mathrm{jet}}>30 \mathrm{GeV}$ and $\left|\mathrm{y}^{\mathrm{jet}}\right|<$ 2.5. In order to improve the precision of the measurement, the results in the $Z \rightarrow e^{+} e^{-}, Z \rightarrow \mu^{+} \mu^{-}$ channels are combined, taking into account correlated systematic uncertainties.

The fiducial production cross sections for $Z$ bosons plus inclusive one to four jet multiplicities have been measured and ratios of the production cross section for successive jet multiplicities have also been derived.

As shown in Figure 9, the measured fiducial cross sections for the various jet multiplicities and the ratios of successive jet multiplicities have also been compared to predictions from SHERPA and from MADGRAPH. The predictions show a reasonable agreement with the observed cross sections and cross section ratios within the uncertainties.

\section{Conclusions}

The analyses presented here cover a range of precision measurements of $W$ and $Z$ production at the ATLAS experiment in proton-proton collisions ranging from a center of mass energy from $7 \mathrm{TeV}$ to 13 $\mathrm{TeV}$. ATLAS has also carried out a wide range of measurements of vector boson with accompanying 


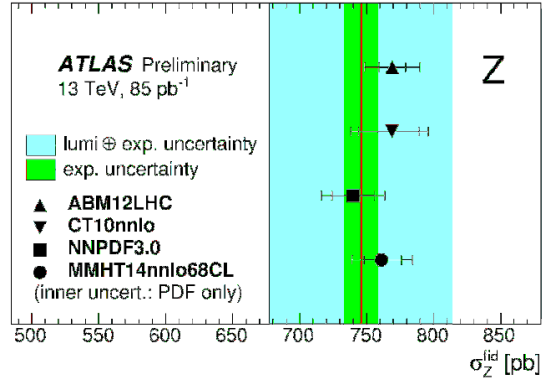

(a)

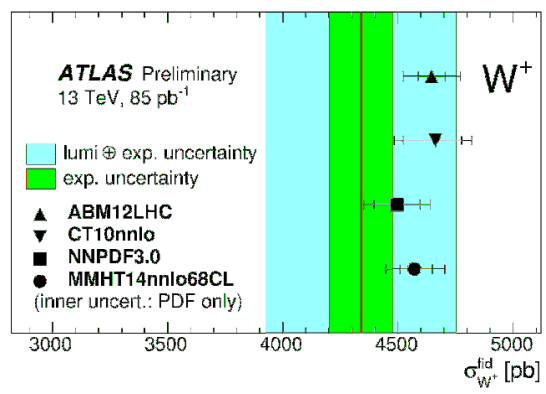

(c)

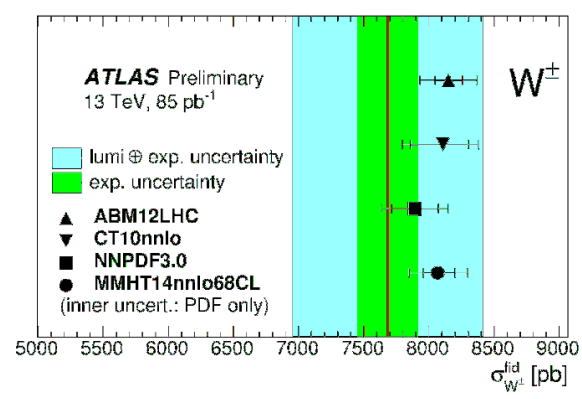

(b)

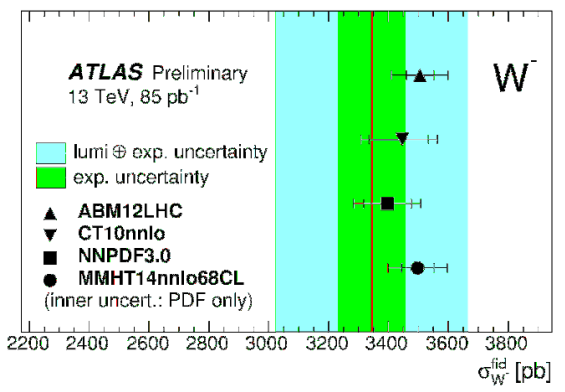

(d)

Figure 7: Predictions for the fiducial cross sections $\sigma_{Z}^{\mathrm{fid}}, \sigma_{W^{ \pm}}^{\mathrm{fid}}, \sigma_{W^{+}}^{\mathrm{fid}}$ and $\sigma_{W^{-}}^{\mathrm{fid}}$ for the four PDFs CT10nnlo, NNPDF3.0, MMHT14nnlo68cl, and ABM12LHC compared to the measured fiducial cross sections (red line). The green (cyan) band corresponds to the experimental uncertainty without (with) the luminosity uncertainty. The inner error bar of the predictions represents the PDF uncertainty while the outer error bar includes the sum of all other systematics. Taken from Ref. [23].

jet production to test and constrain QCD calculations.

All 7 and $8 \mathrm{TeV}$ precision analyses show overall a good agreement with the theory predictions. First studies of the $13 \mathrm{TeV}$ data show a good understanding of the early ATLAS detector performance and are agreeing with the theoretical expectation.

\section{References}

[1] ATLAS Collaboration, JINST 3, S08003 (2008)

[2] ATLAS Collaboration, JHEP 09, 049 (2015)

[3] ATLAS Collaboration, JHEP 09, 145 (2015)

[4] K. Melnikov and F. Petriello, Phys. Rev. D74 114017 (2006)

[5] R. Gavin, Y. Li, F. Petriello, and S. Quackenbush, Comput. Phys. Commun. 182, 2388-2403 (2011)

[6] Y. Li and F. Petriello, Phys. Rev. D86, 094034 (2012)

[7] S. Catani, L. Cieri, G. Ferrera, D. de Florian, and M. Grazzini, Phys. Rev. Lett. 103, 082001 (2009) 


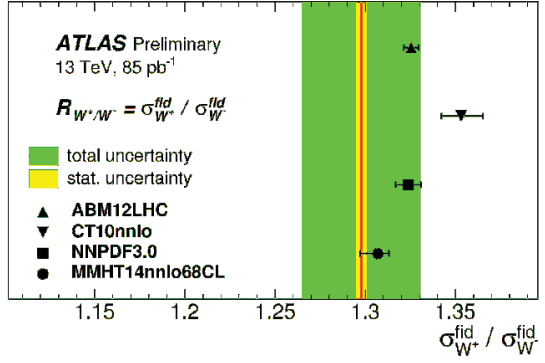

(a)

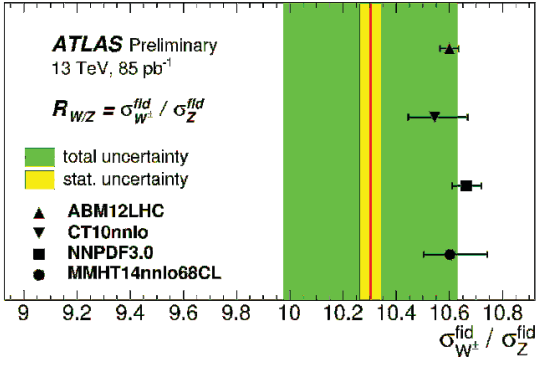

(b)

Figure 8: Ratio of $W^{+}$to $W^{-}$-boson (a) and Ratio of $W^{ \pm}$to $Z$-boson (b) production fiducial cross sections (red line) compared to predictions based on different PDF sets. The inner shaded band corresponds to the statistical uncertainty while the outer band shows statistical and systematic uncertainties added in quadrature. The theory predictions are given with the corresponding PDF uncertainties shown as error bars. Scale uncertainties are not included in the error bars of the predictions. Taken from Ref. [23].

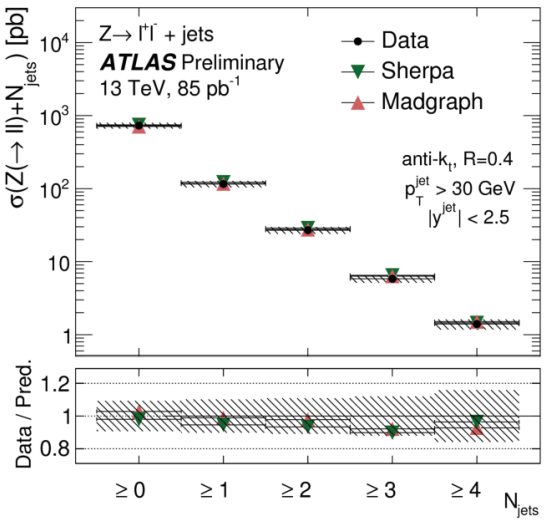

(a)

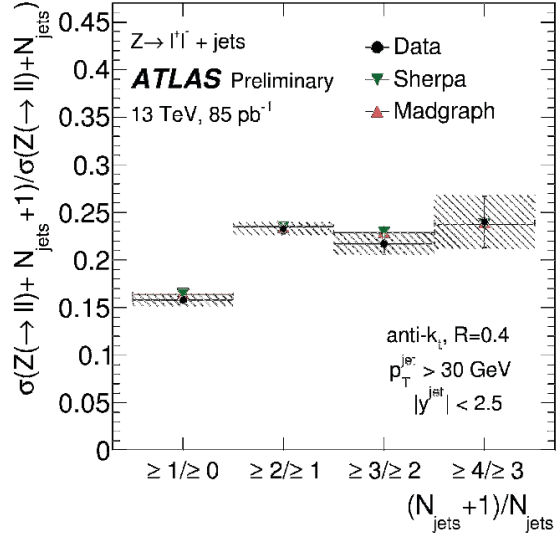

(b)

Figure 9: Combined $Z \rightarrow l^{+} l^{-}$plus $\geq N_{\text {jets }}$ fiducial cross sections (a), and cross section ratios for successive jet multiplicities. Results are reported at the Born level and compared to predictions from Sherpa and MadGraph. The hatched error band corresponds to the total uncertainty of the results: systematic, statistical, and luminosity uncertainties for the fiducial cross section results (a), systematic and statistic uncertainties for the ratio results (b). Taken from Ref. [24].

[8] S. Catani and M. Grazzini, Phys. Rev. Lett. 98, 222002 (2007)

[9] C. Balazs and C. P. Yuan, Phys. Rev. D56, 5558-5583 (1997)

[10] M. Guzzi, P. M. Nadolsky, and B. Wang, Phys. Rev. D90 no. 1, 014030 (2014) 
[11] ATLAS Collaboration, Phys. Lett. B720, 32-51 (2013)

[12] A. Banfi, et al., Eur. Phys. J. C71, 1600 (2011)

[13] ATLAS Collaboration, Eur. Phys. J. C75 no. 2, 82 (2015)

[14] M. L. Mangano, M. Moretti, F. Piccinini, R. Pittau, and A. D. Polosa, JHEP 07, 001 (2003)

[15] T. Gleisberg, S. Hoeche, F. Krauss, M. Schonherr, S. Schumann, F. Siegert, and J. Winter, JHEP 02, 007 (2009)

[16] S. Hoeche, F. Krauss, M. Schonherr, and F. Siegert, JHEP 04, 027 (2013)

[17] J. R. Andersen, T. Hapola, and J. M. Smillie, JHEP 09, 047 (2012)

[18] J. R. Andersen and J. M. Smillie, JHEP 01, 039 (2010)

[19] Z. Bern, L. J. Dixon, F. Febres Cordero, S. Hoeche, H. Ita, D. A. Kosower, D. Maitre, K. J. Ozeren, Phys. Rev. D88 no. 1, 014025 (2013)

[20] C. F. Berger, Z. Bern, L. J. Dixon, F. Febres Cordero, D. Forde, T. Gleisberg, H. Ita, D. A. Kosower, and D. Maitre, Phys. Rev. D80, 074036 (2009)

[21] C. F. Berger, Z. Bern, L. J. Dixon, F. Febres Cordero, D. Forde, T. Gleisberg, H. Ita, D. A. Kosower, and D. Maitre, Phys. Rev. Lett. 106, 092001 (2011)

[22] ATLAS Collaboration, JHEP 07, 032 (2013)

[23] ATLAS Collaboration, ATLAS-CONF-2015-039, (2015)

[24] ATLAS Collaboration, ATLAS-CONF-2015-041, (2015) 